\title{
A Rare Case of ARDS From Human Anaplasmosis
}

\author{
Upendra Kaphle MD, Fayez Kheir MD MSCR, and Supat Thammasitboon MD
}

\begin{abstract}
Human granulocytic anaplasmosis is a tick-borne bacterial disease caused by Anaplasma phagocytophilum. ARDS is a very rare presentation of human anaplasmosis. Early suspicion and empiric antibiotics usually prevent rapid progression of the disease. In our case, despite early initiation of empiric antibiotics, the clinical course of our patient continued to deteriorate but responded dramatically upon addition of steroids. Clinicians should be vigilant about the presentation, diagnostic workup, and treatment of human granulocytic anaplasmosis. Key words: rare diseases; acute respiratory distress syndrome; human anaplasmosis; tick-borne diseases; bacterial infections; antibiotics. [Respir Care 2015;60(7):e125-e127. (c) 2015 Daedalus Enterprises]
\end{abstract}

\section{Introduction}

Human granulocytic anaplasmosis (HGA) is caused by Anaplasma phagocytophilum, an obligate intracellular bacterium that grows within membrane-bound vacuoles in human leukocytes. HGA is an unusual cause of ARDS. We report what is, to our knowledge, the second reported case of HGA complicated by ARDS. Physicians should always consider the possibility of anaplasmosis-related ARDS in patients with unclear, rapid, and worsening respiratory status with a travel history in an endemic area during the summer months.

\section{Case Report}

A 62-y-old man was admitted to the hospital with fever. He had no symptoms until 1 week before hospitalization, when he began complaining about persistent high-grade temperature accompanied with chills, malaise, sweating, and shortness of breath. He had recently traveled to the northeast region of the United States. He denied cough, hemoptysis, rash, insect bites, or sick contacts. His past

The authors are affiliated with the Division of Pulmonary and Critical Care Medicine, Tulane University, New Orleans, Louisiana.

The authors have disclosed no conflicts of interest.

Correspondence: Upendra Kaphle MD, Division of Pulmonary and Critical Care Medicine, Tulane University, 1430 Tulane Avenue, SL-9, New Orleans, LA 70112-2699. E-mail: ukaphle@tulane.edu.

DOI: $10.4187 /$ respcare. 03714 medical history was unrevealing. He was an active 10pack-year smoker. On physical examination, he was diaphoretic but comfortable. Vital signs were within normal limits, other than a temperature of $104^{\circ} \mathrm{F}$. Oxygen saturation was $95 \%$ on room air. His lungs were clear to auscultation bilaterally. The rest of his physical examination was normal.

Blood work revealed a hemoglobin level of $10.2 \mathrm{~g} / \mathrm{dL}$, white blood cell count of 1,900 cells $/ \mu \mathrm{L}$ (neutrophils, $85 \%$; immature granulocytes, $0.4 \%$; and eosinophils, $0 \%$ ), and platelet count of 104,000 cells/ $\mu \mathrm{L}$. The patient's electrolytes, creatinine, and urinalysis were normal. Liver enzymes were normal except for aspartate aminotransferase of $88 \mathrm{U} / \mathrm{L}$ (normal, $<39 \mathrm{U} / \mathrm{L}$ ). He had a C-reactive protein level of $1.13 \mathrm{mg} / \mathrm{dL}$ (normal, $<0.03 \mathrm{mg} / \mathrm{dL}$ ), erythrocyte sedimentation rate of $30 \mathrm{~mm} / \mathrm{h}$ (normal, $0-20 \mathrm{~mm} / \mathrm{h}$ ), and ferritin level of $3,700 \mu \mathrm{g} / \mathrm{L}$ (normal, $23-338 \mu / \mathrm{L})$. The chest radiograph is shown in Figure 1.

The patient was treated empirically with vancomycin, meropenem, and azithromycin. Intravenous doxycycline (100 mg every $12 \mathrm{~h}$ ) was also started in consideration of his recent travel. The patient's condition deteriorated rapidly over the next $24 \mathrm{~h}$. His repeat chest radiograph is shown in Figure 2. The patient was intubated for severe respiratory distress and then ventilated following a lungprotective strategy. The initial ventilator settings were volume assist control, $\mathrm{F}_{\mathrm{IO}_{2}}$ of 1.0, tidal volume of $400 \mathrm{~mL}$ (ideal body weight, $68 \mathrm{~kg}$ ), breathing frequency of 25 breaths/min, and PEEP of $14 \mathrm{~cm} \mathrm{H}_{2} \mathrm{O}$. The corresponding peak and plateau pressures were 32 and $22 \mathrm{~cm} \mathrm{H}_{2} \mathrm{O}$, respectively. The corresponding arterial blood gas results were $\mathrm{pH} 7.20, \mathrm{P}_{\mathrm{CO}_{2}}$ of $53 \mathrm{~mm} \mathrm{Hg}, \mathrm{P}_{\mathrm{O}_{2}}$ of $65 \mathrm{~mm} \mathrm{Hg}$, 


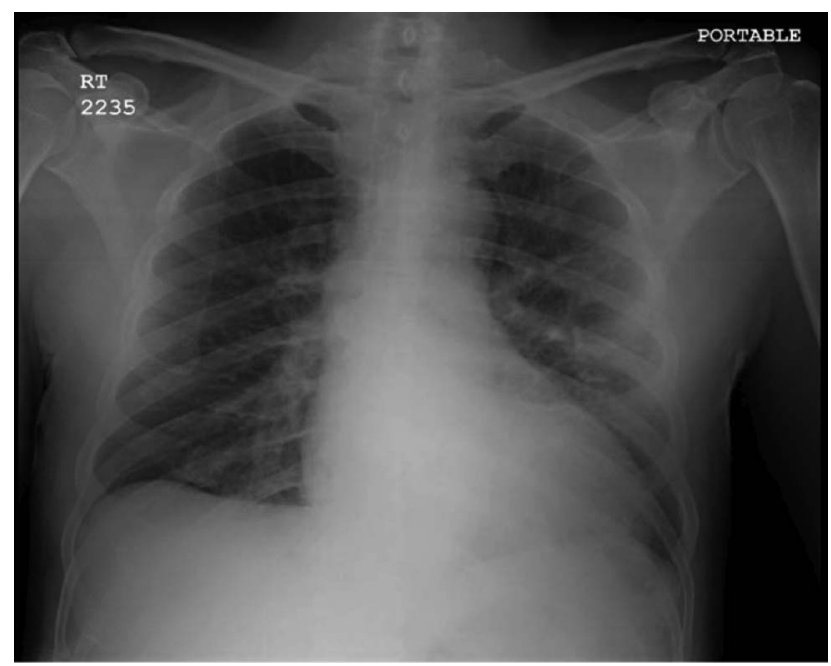

Fig. 1. Chest radiograph showing bilateral linear interstitial abnormality.

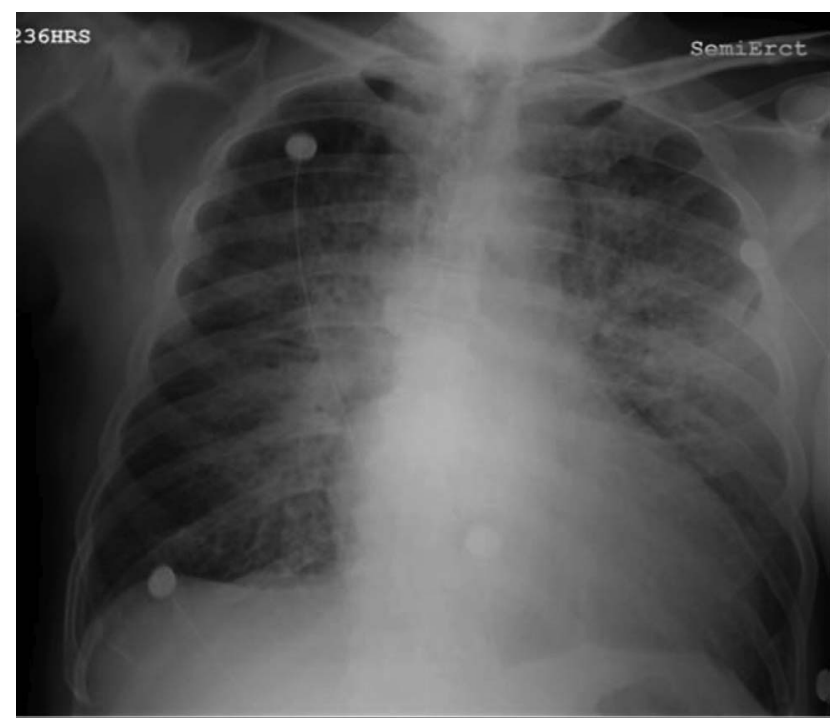

Fig. 2. Follow-up radiograph on day 2 showing increasing lung density, with patches of consolidation.

$\mathrm{HCO}_{3}{ }^{-}$of $21 \mathrm{mmol} / \mathrm{L}$, and $\mathrm{O}_{2}$ saturation of $88 \%$. The ventilator settings were adjusted afterwards following the ARDS Network protocol. The chest computed tomography scan is shown in Figure 3. His blood cultures were negative. Bronchoalveolar lavage was performed on day 2 in the ICU. It was clear, with $73 \%$ macrophages, $20 \%$ lymphocytes, and 7\% neutrophils. Testing for bacterial, viral, and fungal respiratory pathogens was negative. Blood tests for human immunodeficiency virus, hepatitis, fungus, Lyme, Babesia, and Ehrlichia were negative. A peripheral blood smear was sent as an initial workup for tick-borne diseases, which showed morulae (Fig. 4). Confirmatory tests for Anaplasma were done, which showed normal serum immunoglobulin $\mathrm{G}$ and immunoglobulin $\mathrm{M}$, and

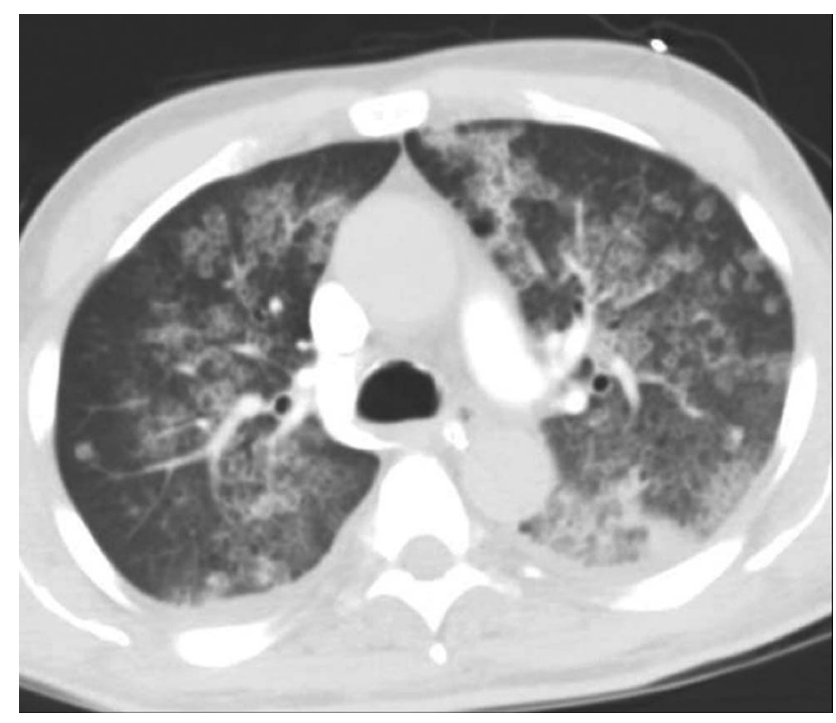

Fig. 3. Computed tomography scan of the chest (lung window) showing bilateral patchy ground-glass opacities.

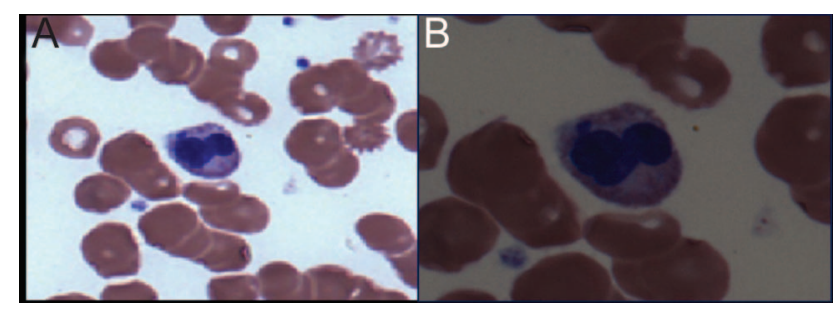

Fig. 4. Peripheral blood smear (Wright stain) showing neutrophils with intracytoplasmic basophilic stippling. A: Original magnification $\times 40$. B: Original magnification $\times 100$.

positive real-time polymerase chain reaction for Anaplasma DNA. Our patient had human anaplasmosis complicated with severe ARDS and some features of macrophage activation syndrome, including cytopenia, elevated ferritin levels, mildly deranged liver enzymes, and fever. Despite early initiation of doxycycline, the clinical condition of our patient continued to deteriorate, with severe hypoxemia. Upon literature review, we found a case report of ehrlichiosis with ARDS for which the addition of steroids resulted in dramatic clinical improvement. ${ }^{1}$ Intravenous methylprednisolone (50 mg 4 times per day) was started on day 2 in the ICU and resulted in dramatic clinical and radiological improvement. The patient was extubated after $72 \mathrm{~h}$, and his clinical condition improved afterwards. The steroid was stopped on day 4 , and he was subsequently discharged home on oral doxycycline.

\section{Discussion}

HGA is caused by A. phagocytophilum. It is an obligate intracellular bacterium that grows within membrane-bound 


\section{A Rare Case of ARDS From Human Anaplasmosis}

vacuoles in human leukocytes. The presentation of HGA is very similar to that of human monocytic ehrlichiosis, which is caused by Ehrlichia chaffeensis and Ehrlichia ewingii. Human monocytic ehrlichiosis and HGA are increasingly recognized as important and frequent causes of fever after tick bite in the Upper Midwest, New England, parts of the Middle Atlantic states, and Northern California..2,3 The main vectors for disease transmission in the United States are Ixodes scapularis and Ixodes pacificus. Typically, the patient presents with fever, headache, myalgia, malaise, leukopenia, thrombocytopenia, and mild liver injury. ${ }^{2,3}$ Frequently, a history of tick bite or bite marks may not be obvious. The clinical features of both anaplasmosis and ehrlichiosis overlap with those of Rocky Mountain spotted fever but typically without rash. Older patients and those with comorbid illnesses or immunosuppressive conditions such as human immunodeficiency virus appear to have more severe infections. Various complications have been reported, such as septic shock-like syndrome, coagulopathy, myocarditis, renal failure, and opportunistic infections. ${ }^{1,4}$ ARDS is a very rare presentation of HGA. Only one case abstract of anaplasmosis with ARDS has been reported in the literature. ${ }^{4}$ The pathophysiology of ARDS in anaplasmosis is unclear. Patients with severe anaplasmosis infection may develop clinical, histopathologic, and laboratory findings that are consistent with many features of macrophage activation and hemophagocytic syndromes. ${ }^{5}$ Various methods have been used to establish the diagnosis of anaplasmosis, including polymerase chain reaction, serological studies, buffy coat examination, and immunohistologic methods. Culture is extremely difficult. Serology using the indirect fluorescent antibody test is the most widely available of these methods. Serologic crossreactivity can occasionally occur between A. phagocytophilum and E. chaffeensis, so it is not possible to reliably differentiate between these 2 infections with a single serologic assay. The indirect fluorescent antibody test has a high sensitivity when obtained $14 \mathrm{~d}$ following the onset of symptoms. Examination of a Wright-stained peripheral blood smear shows intracytoplasmic inclusions (morulae) as stippled blue inclusions of bacteria in neutrophils in $25-75 \%$ of cases and is the most rapid diagnostic method that can be used after disease onset. Polymerase chain reaction amplification of Anaplasma DNA is also available, with a reported sensitivity of $67-90 \% .^{2,6} \mathrm{~A}$ few cases of anaplasmosis have also been diagnosed by staining bone marrow tissue or autopsy tissue. Anaplasmosis is a serious disease with significant morbidity, particularly if appropriate antibiotics are delayed. ${ }^{7}$ Early suspicion and empiric antibiotics usually prevent rapid progression of the disease. The drug of choice in all patients is oral or intravenous doxycycline at $100 \mathrm{mg}$ twice daily for 7-10 d.2,3 Patients who have intolerance or allergy to doxycycline can be treated with rifampin. Based on our anecdotal experience and other case reports of near-fatal ARDS from ehrlichiosis, ${ }^{1}$ it may be reasonable to consider steroid treatment for anaplasmosis with progressive severe ARDS despite treatment with doxycycline. Preventive antibiotic therapy with doxycycline is not recommended. Use of tick repellant remains the only practical preventive measure.

\section{REFERENCES}

1. Patel RG, Byrd MA. Near fatal acute respiratory distress syndrome in a patient with human ehrlichiosis. South Med J 1999;92(3):333335 .

2. Bakken JS, Dumler S. Human granulocytic anaplasmosis. Infect Dis Clin North Am 2008;22(3):433-448.

3. Dumler JS, Madigan JE, Pusterla N, Bakken JS. Ehrlichioses in humans: epidemiology, clinical presentation, diagnosis, and treatment. Clin Infect Dis 2007;45(Suppl 1):S45-S51.

4. Koff G, Sellers J, Oxman D. Anaplasmosis and ARDS. Crit Care Med 2013;41(12):A1231.

5. Dumler JS, Barat NC, Barat CE, Bakken JS. Human granulocytic anaplasmosis and macrophage activation. Clin Infect Dis 2007;45(2): 199-204.

6. Bakken JS, Haller I, Riddell D, Walls JJ, Dumler JS. The serological response of patients infected with the agent of human granulocytic ehrlichiosis. Clin Infect Dis 2002;34(1):22-27.

7. Hamburg BJ, Storch GA, Micek ST, Kollef MH. The importance of early treatment with doxycycline in human ehrlichiosis. Medicine 2008;87(2):53-60. 